\title{
Does representation matter? Teacher-provided tables and drawings as cognitive tools for solving non-routine word problems in primary school.
}

\author{
Timo Reuter ${ }^{1, *}$, Wolfgang Schnotz ${ }^{2}$ and Renate Rasch ${ }^{3}$ \\ 1, * Graduate School “Teaching and Learning Processes", University of Koblenz-Landau, Landau, Germany \\ 2 Department of General and Educational Psychology, University of Koblenz-Landau, Landau, Germany \\ ${ }^{3}$ Institute for Mathematics, University of Koblenz-Landau, Landau, Germany \\ For correspondence: reutertimo@uni-landau.de
}

\begin{abstract}
According to the German educational standards, students should be familiar with problem-solving as a general mathematical competency by the end of grade 4 . Non-routine word problems are suitable tasks for mathematical problem-solving in elementary mathematics classes. They are characterized by the fact that the problemsolver cannot simply use well-trained algorithmic calculating procedures (Rasch, 2001). As a result, many students struggle with word problems in mathematics, especially with non-routine word problems (Hohn, 2012). Representation plays a central role in the process of problem-solving. It involves representing a problem situation adequately, constructing a mental model and comparing it in a dynamic and iterative process with the information externalized in the representation (Schnotz et al, 2011). This study aims to shed light on teacher-provided representations as cognitive tools for students when working on non-routine word problems. In an experimental study, we examined a sample of 67 primary school students who worked on six non-routine word problems with provided representations. The tasks were accompanied by a table or a drawing. Furthermore, the tables and drawings differed with regard to the amount of information provided. Statistical data analysis generated, among other findings, two results: Overall solution rates were low (10 to $24 \%)$. Tables and drawings facilitated the solution process differently depending on the type of word problem. Consequences for subsequent future research are discussed.
\end{abstract}

Keywords: non-routine word problems, external representations, primary students

\section{Introduction}

"A snail in a $24 \mathrm{~m}$ deep well wants to crawl up to the field. Each day it crawls $6 \mathrm{~m}$ up the side of the well and slides down half the distance it crawled in the daytime during the night when sleeping. The snail starts on Monday morning. On which day will the snail reach the top of the well?" This famous riddle is considered to be a non-routine word problem. Non-routine word problems are characterized by the fact that the problem solver cannot simply use well-trained algorithmic calculating procedures (Rasch, 2001, p. 26), but rather has to re-structure existing knowledge to develop a solution (Winter, 1992). In line with Duncker's (1935) classical definition of problem-solving, the student has a goal and does not know how to reach this goal. The given state cannot be transferred into the goal state by simply doing (which would be a task), but requires thinking (Duncker, 1974). Therefore, non-routine word problems provide an ideal object for problem-solving in the primary mathematics classroom. According to the German educational standards, students should be familiar with problem-solving as a general mathematical competency by the end of grade 4 (The Standing Conference of the Ministers of Education and Cultural Affairs of the Länder in the Federal Republic of Germany, 2005, p. 7). Empirical data show that demanding word problems can enhance early mathematical understanding, which is a necessary prerequisite for mathematical competence (Stern, 2005) and, in turn, for later success in mathematics.

What are the cognitive processes when a student works on a mathematical word problem? In the literature on modelling, different authors suggest different modelling cycles (Kaiser, 1995; Kintsch and Greeno, 1985; Mayer and Hegarty, 1996; Nesher, 2003; Reusser, 1997; Verschaffel et al, 2000; for 
an overview see Borromeo-Ferri, 2006). Even though the modelling cycles differ in certain aspects, all process models somehow distinguish between two main parts of the modelling process: representation and solution (Mayer and Hegarty, 1996). In simple terms, representation means that the problem-solver tries to understand the problem, whereas solution means carrying out the necessary operations to solve it (e.g. calculating). The difficulty of solving mathematical word problems is not primarily executing the necessary operations, but understanding the problem for which an adequate situation model has to be constructed (Mayer and Hegarty, 1996; Verschaffel et al, 2000). Mayer and Hegarty (1996) distinguish between two approaches of problem-solvers when working on a word problem: the "problem model strategy" and the "direct translation strategy". When using a "problem model strategy", the problem-solver constructs a situation model, whereas when using a "direct translation strategy" or "superficial strategy" (Verschaffel et al, 2000) she or he skips the situation model and calculates more or less blindly with the numbers given in the text. With regard to the snail problem presented above, the difficulty of the problem does not primarily stem from executing multi-step addition and subtraction without mistakes, but from the need to correctly understand the whole situation described in the text, which requires qualitative reasoning (Mayer and Hegarty, 1996). But even when the upwards and downwards movements are correctly understood, a direct translation strategy such as " 24 meters divided by 3 meters a day $=8$ days" leads to the wrong solution, for the model does not take into consideration that on the last day the snail reaches the top of the well and does not slide back anymore.

How do problem-solvers construct a situational model? The Integrated Text and Picture Comprehension Model (ITPC) of (Schnotz, 2005) assumes that the construction process of a mental model (= situation model) is fed by external sources (e.g. external representations) and internal sources (prior knowledge). External representations can act as cognitive tools in the whole modelling process when solving mathematical (non-)routine word problems. When a problem-solver makes use of external representations, he starts internal communication by producing and receiving signs alternately (Schnotz et al, 2011, p. 217). He compares his mental model (situation model) in a dynamic and iterative process with the information externalized in the representation (Cox, 1999), captures the unstable mental representations and relieves his working memory (Schnotz et al, 2011, p. 217). Schnotz (2002) generally distinguishes between two different types of external representation: descriptive and depictive representations. Descriptive representations consist of symbols with an arbitrary structure that share no resemblance with the object they are representing. On the other hand, depictive representations consist of icons. These icons are signs that always have a spatial configuration and share resemblance or other structural commonalities with the object they are referring to. This gives depictive representations a high inferential efficiency (Schnotz, 2005). To put it simply, a descriptive representation describes something (for example a situation); a depictive representation shows it (Schnotz et al, 2011). The present study examines the usefulness of both descriptive and depictive representations for solving non-routine word problems. Drawings are examined as depictive representations and tables as descriptive representations. Both are relevant in the classroom and can be considered as heuristic tools for solving word problems (Bruder and Collet, 2012). According to the ITPC of Schnotz (2005), the different information sources are processed via different processing routes to construct a mental model. The ITPC distinguishes between the verbal and the pictorial channel. Information from written (or spoken) text is processed by the verbal channel, whereas a picture is processed by the pictorial channel. When reading the text of a word problem, the problem-solver has to select relevant information, organize it in a propositional representation and construct a mental model by activating prior knowledge (Schnotz, 2005). When using a picture as external source of information, the relevant pictorial information has to be selected, organized and again combined with prior knowledge in order to construct a mental model (Schnotz, 2005). As described before, the difficulty of solving word problems is mainly constructing an adequate mental model of the situation described in the problem. Many students seem to have difficulties constructing a mental model of the word problem on the basis of the problem text alone. Especially for children with low prior knowledge as internal source of information, adding a depictive representation to the problem text, which constitutes another source of information processed via a second channel (pictorial channel), should enhance mental model construction (Schnotz, 2005, p. 62). Therefore, a depictive representation must appropriately represent the structure of the problem, regardless of attention is paid to surface details (Pantziara et al, 2009). 
Looking at the snail problem, an appropriate drawing would depict the total distance the snail has to crawl and the upwards and downwards movements. Such a drawing would emphasize the importance of the last day. Although the information is in the text, the problem-solver is more likely to discover it in a picture. Bruder and Collet (2012) strengthen the advantage of a pictorial representation for problem-solving in highlighting the possibility of simply reading off information and relations that are not obvious in the text. The strength of a table, on the other hand, lies in the possibility of structuring the information systematically and clearly (Bruder and Collet, 2012). A table organizing the relevant information like the distance crawled by the snail each day, the distance the snail slipped back each night in combination with the days of the week would allow the problemsolver to systematically work on the problem. However, the table as a descriptive representation is processed via the verbal channel like the word problem text. Therefore no additional channel to construct a mental model can be used. Making sense of the table and working appropriately with it requires the correct mental model of the problem situation beforehand.

\section{Research questions and research hypotheses}

The principle idea of the present study was to provide the students with two different types of representations with non-routine word problems: a drawing (depictive) and a table (descriptive). Furthermore, the drawings and the tables differed in the level of support provided.

The following research questions were adressed:

- Which teacher-provided representation - drawing or table - facilitates the solution process more?

- Which level of support provided in the representations facilitates the solution process more?

The following research hypotheses were examined:

- Hypothesis 1: A drawing enhances the construction of an adequate situation model more than a table, which results in better problem-solving.

- Hypothesis 2: A drawing enables easier access to information, which results in shorter processing time and lower perceived difficulty.

- Hypothesis 3: A higher level of support provided in the representations results in more effective problem-solving, shorter processing time and lower perceived difficulty.

\section{Method}

Participants.

The subjects were 67 pupils (4th grade, 33 female, 32 male, $4 \mathrm{n} / \mathrm{a}$ ) of two primary schools in Germany. Their average age was 8.44 years $(S D=0.636)$.

\section{Experimental design.}

The experiment had a ( $3 \times 2 \times 5)$ factorial design with "type of word problem" as one factor, "type of representation" as another factor and "level of support provided" as third factor. The factor "type of word problem" had three factor levels, namely three types of non-routine word problems: comparison, motion and combinatorics problems. The factor "type of representation" had two factor levels, namely table and drawing. The factor "level of support provided" had five factor levels; the amount of support in the representation increased level by level. We conducted a within-subjects experiment in a multi-matrix design. This meant in practical terms that each participant was administered two test booklets, one after another, each consisting of three word problems (comparison, motion and combinatorics). The word problems were not identical for booklet 1 and booklet 2, but the mathematical structure and numbers were identical. Only the surface of the problems was changed shlightly, e.g. a snail crawling up a well vs. a koala-bear climbing up a tree. One test booklet presented all the word problems together with the one type of representation; the following test booklet presented them together with the other type of representation. To control for order effects, half of the participants started with the drawing-booklet, the other half with the tablebooklet. With regard to the levels of support provided in the representation each participant received three levels. The levels were distributed systematically across the word problems. The booklets were 
randomly distributed to the participants. The dependend variables were the solution rates, processing time and perceived difficulty.

\section{Materials.}

The experiment involved altogether six word problems: two comparison problems, two motion problems and two combinatorics problems. An example of a comparison problem is: "Lukas and Jonas have $18 \mathrm{Yu}$-Gi-Oh cards together. Lukas has 4 cards more than Jonas. How many cards does Lukas have? How many cards does Jonas have?" The difficulty of this type of word problem is the fact that there are two conditions which pupils have to handle simultaneously without being familiar with algebraic solution procedures. An example for a motion problem is the snail problem, which has already been mentioned. An example of a problem involving combinatorics is as follows: "Jonas, Marie, Leoni und Alexander are going on vacation. Each child says goodbye to each of the others with a handshake. How many handshakes is that?" Primary students are not familiar with combinatorics and therefore cannot simply calculate the answer using a routine procedure.

Each word problem was presented with a task-specific drawing or table. As far as possible, the drawing and the corresponding table were informational equivalent (Schnotz, 2002). Figure 1 gives an overview of the representations. For the comparison problem the drawing consisted of appropriately sized rectangles representing known and unknown quantities, using children's knowledge of partwhole-relationships. Bruder and Collet (2012) suggest that whenever an object or a certain number of (unknown) objects has to be divided into parts, it can be visualized by a line or a rectangle. In Singapore this kind of drawing has been taught as "model method" in primary schools since 1983 (Ng and Lee, 2009). The corresponding table consisted of four columns: one column stood for the sum condition and was headed with, e.g. "the cards of Lukas and Jonas together"; another column stood for the difference condition and was headed with, e.g. "Lukas has cards more than Jonas"; the other two collumns stood for the unknown quantities, namely in this example "cards of Lukas" and "cards of Jonas". Depending on the level of support the just mentioned headings and numbers were provided or not provided in the table and depicted or not depicted as rectangles in the drawings, respectively. The drawing representing the motion problem consisted of a vertical arrow representing the distance and direction the object (e.g. the snail) has to move. With increasing amount of support, an appropriate scale was added to the arrow together with small arrows representing movements of the object. These arrows were labelled with the appropriate day, daytime and "start" (e.g. "Monday morning - start") or "finish" (e.g. "Monday evening - finish"). The corresponding table consisted of three columns: The first column listed the days, the second column the point at which the object starts that day in the morning (start) and the third column the point at which the object reaches at the end of the day in the evening (finish). Depending on the level of support provided, the same information was provided or not, as in the corresponding drawing. For the combinatorics problem the drawing depicted stick figure. Depending on the amount of support provided one of the children or all four children were depicted and one or more lines connecting the stick figures were drawn to represent a combination (e.g. handshake). The corresponding table listed the names of the children as headings of the columns and as labellings of the rows. Depending on the level of information only one name or all names were provided in the table and possible combinations were allready crossed or not.

\section{Procedure.}

The experiment was conducted in the classroom. The teachers were asked to provide 90 minutes of lesson time for the experiment. After an introduction by the experimenter the students worked on test-booklet 1 without time constraints. When everybody had finished test-booklet 1 , the participants continued with the second booklet after a short break. The participants wrote with electronic pens which recorded their writing process with exact time-measurements. 


\section{Combinatorics problem}

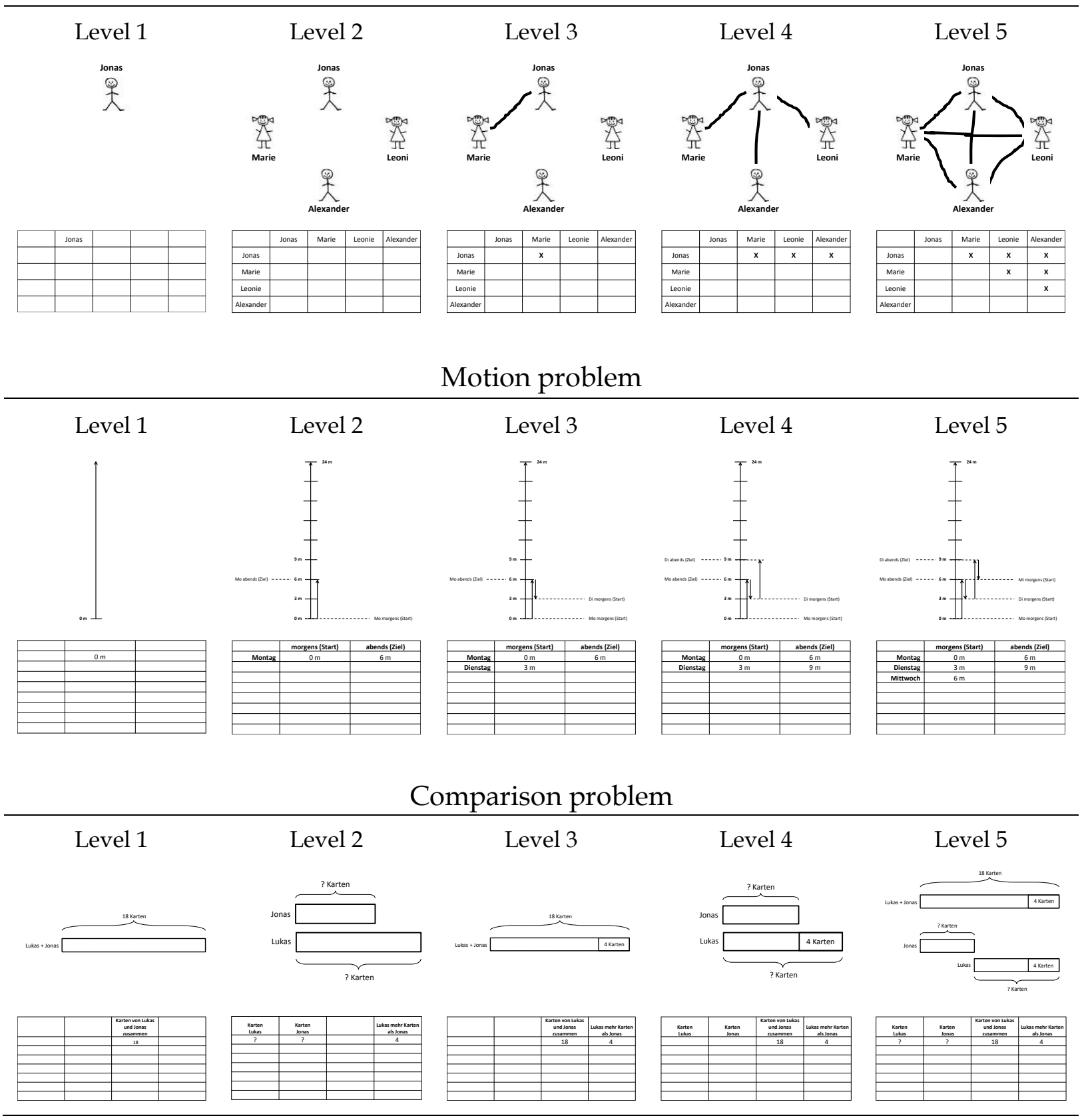

Figure 1. Drawings and tables used for each type of word problem

\section{Results}

The overall solution rates were low. Comparison problem 1 was correctly solved by $16 \%$ of the participants, comparison problem 2 by $22 \%$. Regarding the motion problems, $18 \%$ of the participants provided the correct solution to problem 1 , and $10 \%$ to problem 2 . The combinatorics problems were solved by $15 \%$ (problem 1) of the participants and 12\% (problem 2), respectively. There were no significant differences between the solution rates of the six problems $(Q(5)=5.685, p=0.338)$. Thus, problems 1 and 2 of each type of word problem can be considered equally difficult.

The processing time was measured via the digital recording of the electronic pen for each of the word problems and individually for each participant. The time at which the participant started to read the problem text marked the start and the time at which she or he wrote down the answer marked the end of the processing time interval. The average processing time differed significantly for the six 
problems, $\chi^{2}(5)=82.960, p=0.000$. Pairwise comparisons of the problems sharing the same mathematical structure using the Wilcoxon signed-rank test revealed significant differences. The average processing times for all problems in test booklet 2 were shorter than those for the corresponding problems in test booklet 1 . On average, the participants worked $\bar{x}=288.346$ seconds $(S D=199.768)$ on motion problem 1 , but only $\bar{x}=155.830$ seconds $(S D=148.302)$ on motion problem $2(\mathrm{Z}=-3.862, p=0.000)$. The average processing times for the comparison problems were $\overline{\mathrm{X}}=246.458$ seconds $(S D=157.303)$ and $\bar{x}=84.160(S D=71.399)$ seconds, respectively $(Z=-5.369, p=0.000)$. The processing times for the combinatorics problems were shortest: $\overline{\mathrm{x}}=125.047$ seconds $(S D=125.373)$ for problem 1 and $\bar{X}=53.981$ seconds $(S D=41.745)$ for problem 2 , respectively $(Z=-4.296, p=0.000)$.

The perceived difficulty was rated by the participants on a 4-point Likert scale with $1=$ very easy, $2=$ rather easy, $3=$ rather difficult, $4=$ very difficult directly after they had worked on the problem. The perceived difficulty of the six problems differed significantly, $\chi^{2}(5)=59.594, p=0.000$. Pairwise comparisons showed that combinatorics problem 1 was perceived to be significantly easier than comparison problem $1(Z=-5.100, p=0.000)$ and motion problem $1(Z=-5.096, p=0.000)$. The same applies for combinatorics problem 2 compared to comparison problem $2(Z=-5.427, p=0.000)$ and motion problem $2(Z=-5.383, p=0.000)$. With the exception of the combinatorics problems, the perceived difficulty of the problems in test booklet 2 was significantly lower than that for the corresponding problems in test booklet 1 . The perceived difficulty of motion problem 1 was $\bar{x}=2.75$ $(S D=1.04)$, and $\bar{x}=2.37(S D=1.13)$ for motion problem $2(\mathrm{Z}=-2.520, \mathrm{p}=0.012)$. The perceived difficulty of comparison problem 1 was $\overline{\mathrm{x}}=2.53(S D=0.98)$ and $\overline{\mathrm{x}}=2.16(S D=1.20)$ for comparison problem 2 , respectively $(Z=-2.341, p=0.019)$. The perceived difficulty for combinatorics problem 1 ( $\overline{\mathrm{X}}=1.69, S D=0.70)$ and combinatorics problem $2(\overline{\mathrm{x}}=1.70, S D=0.91)$ did not differ $(\mathrm{Z}=-0.152, \mathrm{p}=$ 0.879). Comparing the solution rates (observed difficulty) with the average perceived difficulty, it seems that the participants tended to underestimate the difficulty of the problems.

Research hypothesis 1 claimed that a drawing facilitates problem-solving more than a table. Therefore the solution rates should be higher when a problem is presented with a drawing. This hypothesis received only partial support from the results. For the combinatorics problem, the drawing significantly contributed to the solution rate. When the problem was presented with a drawing, $19 \%$ of the participants came to the correct solution, whereas only $8 \%$ provided the correct answer when the problem was presented with a table $(Z=-2.530, p=0.011)$. There were no significant differences in the solution rates for the comparison $(Z=-1.633 p=0.102)$ and the motion problems $(Z=-0.905, p=$ $0.366)$ when they were accompanied by either a drawing or a table. Contrary to research hypothesis 1 , the solution rates for the comparison and the motion problems were somewhat higher when the problems were presented with a table: $22 \%$ of the participants provided the correct answer to the comparison problem when presented with a table and $16 \%$ when a drawing was given as external representation. The motion problem was solved correctly by $16 \%$ of the participants when presented with a table and by $12 \%$ when presented with a drawing.

The solution rates of a problem presented with a drawing highly correlated with the solution rates of the corresponding problem presented with a table. Participants who provided either the correct or incorrect answer to the comparison problem presented with a drawing did the same when the problem was presented with a table $\left(r_{\text {tet }}=0.941\right)$. The correlation was $r_{\text {tet }}=0.771$ for the combinatorics problem and $\mathrm{r}_{\text {tet }}=0.588$ for the motion problem.

Table 1. Solution rates: Comparison of drawing and table.

\begin{tabular}{lcc}
\hline & \multicolumn{2}{c}{ Type of representation } \\
\cline { 2 - 3 } Type of non-routine word problem & $\begin{array}{c}\text { Drawing } \\
(\mathrm{n}=67)\end{array}$ & $\begin{array}{c}\text { Table } \\
(\mathrm{n}=67) \\
\%\end{array}$ \\
& 16 & 22 \\
\hline Comparison problem & 12 & 16 \\
Motion problem & 19 & 8 \\
\hline
\end{tabular}


Hypothesis 2 assumed a shorter processing time when the problem is presented with a drawing than when presented with a table. This hypothesis was not supported by the current data. When looking at the processing time data, it is necessary to differentiate between participants who provided the correct answer and those who did not. It is quite difficult to interpret the processing time of students who failed to provide a correct answer. Some of them might have taken quite a long time due to the fact that they did not give up, but finally came to an incorrect answer or even to no answer. Participants who misunderstood the problem might have provided an incorrect answer or no answer very quickly. Thus, in order to draw meaningful conclusions, only participants who provided the correct answers were taken into account when analyzing processing time. Given the low overall solution rates, this led to a small number of participants being analyzed (on average ten persons for each problem). Thus, no statistical tests for significance were conducted. The results reported only give an impression of a tendency and, therefore, have to be treated cautiously.

For all three types of problems, the results showed a longer processing time when the problem was presented with a drawing. This is particularly clear for the motion problem and the combinatorics problem. Participants who provided the correct answer to the motion problem presented with a drawing $(n=8)$ worked $\bar{x}=436.750$ seconds $(S D=240.616)$ on it, whereas participants who provided the correct answer to this problem presented with a table $(n=10)$ only worked $\bar{x}=311.500$ seconds $(S D=195.316)$. Students who found the correct answer for the combinatorics problem presented with a drawing $(\mathrm{n}=13)$ worked $\overline{\mathrm{x}}=114.230$ seconds on it $(S D=111.426)$ and students reaching the correct solution for the combinatorics problem presented with a table $(n=3)$ only worked $\bar{x}=39.900$ seconds $(S D=22.143)$. The comparison problem reveals the same pattern, although the time difference is lower. Participants who were provided with a drawing and came up with the correct answer $(n=10)$ worked $\overline{\mathrm{x}}=168.300$ seconds $(S D=162.120)$ on the problem, whereas participants who were provided with a table and answered correctly $(n=11)$ only worked $\bar{x}=144.100$ seconds $(S D=86.829)$.

Hypothesis 2 also assumed a lower perceived difficulty by the problem-solvers when the word problem is presented with a drawing. This hypothesis received partial support from the data. Again, we only took into account the data of participants who provided the correct answers. This led again to a small number of cases being analyzed. No statistical tests for significance were conducted. Thus, the findings should be treated cautiously. Nevertheless, they showed a clear pattern. A word problem presented with a drawing was perceived to be less difficult by participants who provided the correct answer than when presented with a table. Students who provided the correct answer rated the perceived difficulty of the motion problem to be $\bar{x}=2.00(S D=1.07)$ when presented with a drawing $(\mathrm{n}=8)$ and rated it to be $\overline{\mathrm{x}}=2.45(S D=1.04)$ when presented with a table $(\mathrm{n}=11)$. The comparison problem was rated to be $\bar{x}=1.73(S D=0.90)$ when a drawing was provided $(n=11)$ and $\bar{x}=2.13(S D$ $=1.06)$ with a table $(n=15)$. The perceived difficulty for the combinatorics problem was nearly the same: $\bar{x}=1.54(S D=0.78)$ for the drawing $(n=13)$ and $\bar{x}=1.40(S D=0.55)$ for the table $(n=4)$.

Hypothesis 3 assumed that the higher the level of support provided in the representation, the higher the solution rates would be. This hypothesis received no support. No significant differences in the solution rates were observed for the varying level of information provided, $\chi^{2}(4)=5.738, p=0.220$ (comparison problem with drawings), $\chi^{2}(4)=5.496, p=0.240$ (comparison problem with tables), $\chi^{2}(4)$ $=3.875, p=0.423$ (motion problem with drawings), $\chi^{2}(4)=3.696, p=0.449$ (motion problem with tables), $\chi^{2}(4)=3.441, p=0.487$ (combinatorics problem with drawings), and $\chi^{2}(4)=6.134, p=0.189$ (combinatorics problem with tables). The data did not show a clear pattern (see Table 2), except for the presentation of the combinatorics problem with a drawing. Indeed, $29 \%$ of the participants working on the combinatorics problem with the drawing with the least amount of support $(n=14)$ provided the correct answer. Similarly, $31 \%$ of the participants who were given the problem with the drawing with the most amount of support $(n=13)$ came up with the correct solution. Compared with that, participants who were provided with drawings with a medium amount of support succeeded less. Their solution rates were $7 \%$ for level 2 and $15 \%$ for levels 3 and 4 . This data pattern suggests a curvilinear correlation. The table showed a similar pattern: $21 \%$ of the participants who worked on the combinatorics problem presented with the table with the least amount of support $(n=13)$ provided the correct solution. None of the participants working on the problem with the level $2(n=14)$ and level 3 tables $(n=13)$ provided the correct answer, whereas $8 \%$ of the students came up with the 
correct solution when the level 4 table $(n=14)$ and the level 5 table $(n=13)$ were presented. However, the table data should be treated very cautiously due to the low overall solution rate $(8 \%)$ of the combinatorics problem when a table was presented as external representation.

Looking at the motion problem, we can conclude that a drawing providing more support seems to be more helpful than one with less, suggesting that hypothesis 2 might hold. Interestingly, this is not true for the table. On the other hand, the solution rates for the comparison problem when a drawing was provided suggest an opposite trend to hypothesis 2 . None of the participants who worked on the problem presented with the drawing providing the most support $(n=13)$ reached the correct answer. However, $31 \%$ and $21 \%$ of the students who worked on the problem with drawings providing low support (levels 1 and 2) provided the correct answer. A similar pattern was found for the table. The assumptions regarding the processing time and the perceived difficulty depending on the level of support provided unfortunately could not be tested on this dataset due to the small number of participants who provided a correct answer.

Table 2. Solution rates: Comparison of drawing and table with different levels of support provided

\begin{tabular}{|c|c|c|c|c|c|c|c|c|c|c|c|c|}
\hline \multirow{4}{*}{$\begin{array}{l}\text { Level of } \\
\text { support } \\
\text { provided }\end{array}$} & \multicolumn{12}{|c|}{ Type of non-routine word problem } \\
\hline & \multicolumn{4}{|c|}{ Comparison problem } & \multicolumn{4}{|c|}{ Motion problem } & \multicolumn{4}{|c|}{ Combinatorics problem } \\
\hline & \multicolumn{2}{|c|}{ Drawing } & \multicolumn{2}{|c|}{ Table } & \multicolumn{2}{|c|}{ Drawing } & \multicolumn{2}{|c|}{ Table } & \multicolumn{2}{|c|}{ Drawing } & \multicolumn{2}{|c|}{ Table } \\
\hline & $\%$ & $n$ & $\%$ & $n$ & $\%$ & $n$ & $\%$ & $n$ & $\%$ & $n$ & $\%$ & $n$ \\
\hline 5 & 0 & 13 & 8 & 13 & 14 & 14 & 14 & 14 & 31 & 13 & 8 & 13 \\
\hline 4 & 21 & 14 & 29 & 14 & 23 & 13 & 8 & 13 & 15 & 13 & 8 & 14 \\
\hline 3 & 8 & 13 & 8 & 13 & 0 & 14 & 21 & 14 & 15 & 13 & 0 & 13 \\
\hline 2 & 21 & 14 & 36 & 14 & 15 & 13 & 31 & 13 & 7 & 14 & 0 & 14 \\
\hline 1 & 31 & 13 & 31 & 13 & 8 & 13 & 8 & 13 & 29 & 14 & 21 & 14 \\
\hline
\end{tabular}

Summarizing the results so far, hypothesis 1 which assumed that a drawing would facilitate problemsolving more than a table and would result in higher solution rates, shorter processing time and lower perceived difficulty received only partial support. Higher solution rates for a problem presented with a drawing were only found for the combinatorics problem. Contrary to hypothesis 1 (but based on a small number of participants and therefore to be interpreted cautiously), processing time was higher when the problems were presented with a drawing than with a table. However, perceived difficulty was somewhat lower when the problems were presented with a drawing, which is in line with hypothesis 1.

\section{Discussion and conclusions}

Even though the word problems were presented with external representations, namely a drawing or a table, the overall solution rates were low. Contrary to our hypothesis, the drawing facilitated problem-solving only for one type of problem, namely the combinatorics problem. One reason might be that the participants did not understand the drawings and tables. Qualitative data support this assumption. The participants were asked if the drawing (respectively the table) was useful directly after each word problem in the test booklet. Only $22 \%$ stated that the drawings presented with the comparison problem as useful. On the other hand, $43 \%$ rated the drawings accompanying the motion problem as useful and half of the participants (50\%) considered the drawings presented with the combinatorics problem useful. Almost the same figures were found for the table. In an open-ended question, the participants were asked to state their reasons. Most of the participants who perceived the drawings as not useful claimed that they did not understand them. Many participants also explained that they preferred to calculate mentally. This is in line with findings of studies on strategy use and self-generated representations when solving (non-) routine word problems (De Bock et al, 1998; Elia et al, 2009; Groß, 2013; Hohn, 2012; Verschaffel et al, 1999). It also explains why the table as a heuristic tool was not perceived as useful by most of the participants. This suggests that we need to 
take a closer look at the representations we used with the different types of non-routine word problems. The drawings differed in their level of abstraction. The drawing accompanying the combinatorics problem was the most concrete, depicting at least one child (information level 1) or all four children described in the problem text. Although the depicted children were schematized (stick figures) the participants succeeded in finding the protagonists of the word problem in the drawing. However, the drawings used for the motion and the comparison problems were far more abstract. The drawing accompanying the motion problem did not depict the snail and the well, but was made up of arrows representing the distances and movements. The drawing for the comparison problem did not depict the YU-GI-OH-cards described in the word problem text, but was made up of rectangles representing a certain quantity of cards. A basic principle in multimedia learning is the coherence principle (Mayer, 2005; Schnotz, 2005, p. 60), which states "that a text and a picture can only contribute to the joint construction of the same mental model if the text and the picture are semantically related." (Schnotz, 2005, p. 60). It might have been difficult for primary students to make referential connections between the texts and drawings when no concrete objects described in the texts were depicted in the drawings. Making such referential connections is even difficult for older students (Ainsworth, 1999). In consequence, participants might have not used the drawing at all or misunderstood it. Our participants in the present study did not receive any training on how to work with the representations, which might also explain the low solution rates and the low perceived usefulness of the drawings and tables.

The processing times for all word problems in test booklet 2 were significantly shorter than the processing times for the word problems in test booklet 1 . We explain this finding with methodical reasons and not content reasons. The participants worked on test booklet 2 immediately after they had finished booklet 1 . Contrary to research findings that novices are more likely to stick to the surface details of a problem than experts (Chi et al, 1981), many participants might have realized that the mathematical structure was identical and they therefore reproduced their (incorrect) answers from memory without any further reasoning. This was facilitated by the fact that the two corresponding word problems had the same numbers and quantities. With regard to hypothesis 2, assuming that processing time should be shorter when the problem is presented with a drawing, we found a tendency that processing time was longer when the problem was presented with a drawing. As a possible explanation, we assume that the participants who provided the correct answer when a table was presented, already had a correct situation model, whereas participants who provided the correct answer when a drawing was presented, used this drawing to first construct a situation model which took them more time.

Regarding the level of support provided in the representations no clear results were observed. The straightforward assumption that the higher the support provided, the better the problem-solving, needs to be re-considered and differentiated for the different types of problems. The Generative Theory of Drawing Construction by Van Meter and Garner (2005) suggests that presenting a drawing does not always lead to sufficient coherence-building. However, generating a drawing activates underlying cognitive processes more and enhances meta-cognitive processes. Empirical findings show that generating a picture is useful for learning, but only if the quality of the generated drawing meets the requirements of the task and does not present the learner with too much additional cognitive load (Van Meter and Garner, 2005). With regard to our question the drawing has to appropriately represent the mathematical structure of the problem and sufficiently allow for students to work with it actively.

Subsequent studies should test drawings which are more concrete and also pay attention to the personal variables of the participants (e.g. reading and calculation skills, general cognitive and metacognitive skills, attitudes towards problem solving). To control for prior-knowledge, a premeasurement in which non-routine word problems are presented without providing any representations should be conducted. After the students worked on problems with provided representations, a post-measurement in which again no representations are provided, could investigate for transfer effects. 


\section{References}

Ainsworth, S. (1999). The functions of multiple representations. Computers $\mathcal{E}$ Education, 33(2-3), 131-152.

Borromeo Ferri, R. (2006). Theoretical and empirical differentiations of phases in the modelling process. Zentralblatt für Didaktik der Mathematik 38(2), 86-95.

Bruder, R. and Collet, C. (2011). Problemlösen lernen im Mathematikunterricht. Berlin: Cornelsen Scriptor Praxis.

Chi, M. T. H., Feltovich, P. J., and Glaser, R. (1981). Categorization and Representation of Physics Problems by Experts and Novices. Cognitive Science 5(2), 121-152.

Cox, R. (1999). Representation construction, externalised cognition and individual differences. Learning and Instruction 9(4), 343-363.

De Bock, D., Verschaffel, L. and Janssens, D. (1998). The predominance of the linear model in secondary school students' solutions of word problems involving length and area of similar plane figures. Educational Studies in Mathematics 35(1), 65-83.

Duncker, K. (1974). Zur Psychologie des produktiven Denkens. Berlin, Heidelberg, New York: Springer.

Elia, I., Van den Heuvel-Panhuizen, M., and Kolovou, A. (2009). Exploring strategy use and strategy flexibility in non-routine problem solving by primary school high achievers in mathematics. ZDM Mathematics Education 41(5), 605-618.

Hohn, K. (2012). Gegeben, gesucht, Lösung? Selbstgenerierte Repräsentationen bei der Bearbeitung problemhaltiger Textaufgaben. PhD thesis. Koblenz-Landau, Landau. Psychologie.

Kaiser, G. (1995). Realitätsbezüge im Mathematikunterricht - Ein Überblick über die aktuelle und historische Diskussion. In G. Graumann et al (Eds.), Materialien für einen realitätsbezogenen Mathematikunterricht (pp. 66-84). Bad Salzdetfurth: Franzbecker.

Kintsch, W. and Greeno, J. G. (1985). Understanding and solving word arithmetic problems. Psychological Review 92(1), 109-129.

Mayer, R. E. (2005). Principles for Reducing Extraneous Processing in Multimedia Learning: Coherence, Signaling, Redundancy, Spatial Contiguity, and Temporal Contiguity Principles. In R. E. Mayer (Ed.), The Cambridge handbook of multimedia learning (pp. 183-200). Cambridge, U.K, New York: Cambridge University Press.

Mayer, R. E.; Hegarty, M. (1996). The Process of Understanding Mathematical Problems. In R. Sternberg and T. Ben-Zeev (Eds.), The Nature of Mathematical Thinking (pp. 29-53). Mahwah, NJ: L. Erlbaum Associates.

Nesher, P., Hershkowitz, S. and Novotna, J. (2003). Situation model, text base and what else? Factors affecting problem solving. Educational Studies in Mathematics 52(2), 151-176.

$\mathrm{Ng}$, E. L. and Lee, K. (2009). The Model Method: Singapore Children's Tool for Representing and Solving Algebraic Word Problems. Journal for Research in Mathematics Education 40(3), 282-313.

Pantziara, M., Gagatsis, A. and Elia, I. (2009). Using diagrams as tools for the solution of non-routine mathematical problems. Educational Studies in Mathematics 72(1), 39-60.

Schnotz, W. (2002). Towards an Integrated View of Learning From Text and Visual Displays. Educational Psychology Review 14(1), 101-120.

Schnotz, W. (2005). An Integrated Model of Text and Picture Comprehension. In: R. E. Mayer (Ed.), The Cambridge handbook of multimedia learning (pp. 49-69). Cambridge, U.K, New York: Cambridge University Press.

Schnotz, W., Baadte, C., Müller, A., and Rasch, R. (2011). Kreatives Problemlösen mit bildlichen und beschreibenden Repräsentationen. In K. Sachs-Hombach and R. Totzke (Eds.), Bilder - Sehen - Denken. Zum Verhältnis von begrifflichenphilosophischen und empirisch-psychologischen Ansätzen in der bildwissenschaftlichen Forschung (pp. 204-252). Köln: Herbert von Halem Verlag.

Stern, E. (2005). Kognitive Entwicklungspsychologie des mathematischen Denkens. In M. von Aster and J. H. Lorenz (Ed.), Rechenstörungen bei Kindern. Neurowissenschaft, Psychologie, Pädagogik (pp. 137-149). Göttingen: Vandenhoeck \& Ruprecht.

Van Meter, P. and Garner, J. (2005). The Promise and Practice of Learner-Generated Drawing: Literature Review and Synthesis. Educ Psychol Rev 17(4), 285-325.

Verschaffel, L., De Corte, E., Lasure, S., Van Vaerenbergh, G., Bogaerts, H. and Ratinckx, E. (1999). Learning to solve mathematical application problems: A design experiment with fifth graders. Mathematical Thinking and Learning 1(3), 195-229.

Verschaffel, L., Greer, B., and De Corte, E. (2000). Making sense of word problems. Lisse, Exton, PA: Swets \& Zeitlinger Publishers.

Winter, H. (1992). Sachrechnen in der Grundschule. Problematik des Sachrechnens, Funktionen des Sachrechnens, Unterrichtsprojekte. Berlin: Cornelsen Scriptor 